\title{
Invasive Group A Streptococcal Disease in Ireland, 2004 to 2010
}

J Martin (Jennifer_martin@health.gov.ie) ${ }^{1}$, S Murchan'1, D O’Flanagan'1 , F Fitzpatrick ${ }^{1,2}$

1. Health Protection Surveillance Centre, Dublin, Ireland

2. Department of Clinical Microbiology, Beaumont Hospital, Dublin, Ireland

Invasive group A streptococcal infections (iGAS) are a major clinical and public health challenge. iGAS is a notifiable disease in Ireland since 2004. The aim of this paper is to describe the epidemiology of iGAS in Ireland for the first time over the seven-year period from 2004 to 2010. The Irish national electronic infectious disease reporting system was used by laboratories to enter the source of iGAS isolates, and by departments of public health to enter clinical and epidemiological details. We extracted and analysed data from 1 January 2004 to 31 December 2010. Over the study period, 400 iGAS cases were notified. The annual incidence of iGAS doubled, from 0.8 per 100,000 population in 2004 to 1.6 in 2008, and then remained the same in 2009 and 2010. The reported average annual incidence rates were highest among children up to five years of age $(2.3 / 100,000)$ and adults aged over 60 years $(3.2 / 100,000)$. The most common risk factors associated with iGAS were skin lesions or wounds. Of the 174 people for whom clinical syndrome information was available, 28 (16\%) cases presented with streptococcal toxic shock syndrome and 19 (11\%) with necrotising fasciitis. Of the 141 cases for whom seven-day outcomes were recorded, 11 people died with iGAS identified as the main cause of death (seven-day case fatality rate $8 \%$ ). The notification rate of iGAS in Ireland was lower than that reported in the United Kingdom, Nordic countries and North America but higher than southern and eastern European countries. The reasons for lower notification rates in Ireland compared with other countries may be due to a real difference in incidence, possibly due to prescribing practices, or due to artefacts resulting from the specific Irish case definition and/or low reporting in the early stages of a new surveillance system. iGAS disease remains an uncommon but potentially severe disease in Ireland. Ongoing surveillance is required in order to undertake appropriate control measures and gain a greater understanding of this disease.

\section{Introduction}

Invasive group A streptococcal infection (iGAS) occurs when Streptococcus pyogenes invades a normally sterile site, e.g. blood, cerebrospinal fluid (CSF) or pleural fluid, and is associated with severe disease including necrotising fasciitis (NF), meningitis and streptococcal toxic shock syndrome (STSS) [1].

Although iGAS is relatively uncommon, the rapidity with which patients deteriorate, its occurrence in otherwise healthy people and the difficulties in the differential diagnosis underlie the importance of surveillance of this disease. Surveillance enables early detection of clusters/outbreaks to ensure prompt implementation of infection prevention, and control precautions and appropriate management of contacts. In addition, it allows trends in iGAS to be monitored, to inform healthcare planning and to support ongoing scientific research into, transmission, risk factors, pathogenesis and control of iGAS.

In Ireland, iGAS is a notifiable disease under the Infectious Diseases Regulations 1981. Under Section 14 of these regulations, as amended by S.I. No. 707 of 2003, a medical practitioner and a clinical director of a diagnostic laboratory, on suspecting or identifying a case of the infection, is obliged to send a written or electronic notification to a medical officer of health $(\mathrm{MOH})$ [1]. The $\mathrm{MOH}$ will then undertake/delegate an investigation to identify contacts in order to provide information and prescribe chemoprophylaxis if indicated. Indications are (i) close contacts if they have symptoms suggestive of localised group $A$ streptococcal (GAS) infection or (ii) mother and baby if either develops iGAS in the neonatal period (first 28 days of life). The MOH investigation is also to identify outbreaks.

Following a report of a cluster of cases in the west of the country in early 2005 [2], enhanced surveillance, which includes collection of information on isolate site, clinical presentation, risk factors and patient outcome, has been conducted on iGAS cases, on a voluntary basis, by departments of public health and microbiology laboratories since 2005 . The national electronic infectious disease reporting system (Computerised Infectious 
Diseases Reporting system, CIDR), which allows realtime exchange of information between laboratories, regional departments of public health and the national centre for communicable disease surveillance (Health Protection Surveillance (entre, HPSC), is used to notify iGAS and record enhanced surveillance findings.

Collection of antimicrobial susceptibility data commenced in 2008. These data are collected separately via the national antimicrobial resistance surveillance system.

The aim of this paper is to describe the epidemiology of iGAS in Ireland for the first time over the sevenyear period from 2004 to 2010 in order to improve the understanding of iGAS in Ireland and to compare the epidemiology in Ireland with other countries.

\section{Methods}

\section{Case definition}

Confirmed iGAS cases are defined as patients with S. pyogenes isolated from a normally sterile site (e.g. blood, CSF, pleural fluid). Probable cases are defined as patients with $S$. pyogenes isolated from a non-sterile site (e.g. throat, vagina) combined with a clinical presentation compatible with STSS.

STSS is defined as hypotension (fifth percentile of systolic blood pressure in children, or $490 \mathrm{mmHg}$ systolic pressure in adolescents and adults) and two or more of the following: renal impairment (creatinine greater than twice the upper limit of normal for age), coagulopathy (platelets $1100,000 \times 10^{6} / \mathrm{l}$ or evidence of disseminated intravascular coagulation), liver dysfunction (alanine transaminase, aspartate aminotransferase or bilirubin more than twice the upper limit of normal for age), adult respiratory distress syndrome (pulmonary infiltrates and hypoxaemia without cardiac failure or generalised oedema), generalised erythematous rash that may desquamate or soft tissue necrosis (necrotising fasciitis, myositis or gangrene).

\section{Dataset and data processing}

The iGAS isolate site was entered into CIDR by microbiology laboratories. Clinical details, including seven-day outcome data, and epidemiological details, including clusters of infection, were collected by public health staff and microbiologists through contact with the clinicians caring for the cases, or the cases themselves if they were well enough. Where death occurred, iGAS was attributed as main cause or contributory cause based on clinician's assessment. A cluster was defined as two or more epidemiologically linked iGAS cases or where the observed number of iGAS cases exceeds the expected number [1]. Information was entered into CIDR by public health staff using the national iGAS infection enhanced data form [1]. For laboratories and departments of public health not on CIDR, data were sent to the HPSC where they were entered into the system.

Antimicrobial susceptibility data were collected separately via the national antimicrobial resistance surveillance system.

\section{TABLE 1}

Number of cases of invasive group A streptococcal infection, and Irish age-specific incidence rates per 100,000 aged-matched population, by five year age groups, and calendar year, Ireland, 2004-2010 ( $n=400)$

\begin{tabular}{|l|c|c|c|c|c|c|c|c|}
\hline \multirow{2}{*}{ Age interval in years } & \multicolumn{7}{|c|}{ Number of cases (age-specific incidence rate per 100,000 age-matched population) } \\
\cline { 2 - 9 } & 2004 & 2005 & 2006 & 2007 & 2008 & 2009 & 2010 & $2004-2010$ \\
\hline $0-4$ & $4(1.3)$ & $4(1.3)$ & $7(2.3)$ & $13(4.3)$ & $5(1.7)$ & $9(3)$ & $6(2)$ & $48(2.3)$ \\
\hline $5-9$ & $3(1)$ & $2(0.7)$ & $1(0.3)$ & $4(1.4)$ & $7(2.4)$ & $1(0.3)$ & $4(1.4)$ & $22(1.1)$ \\
\hline $10-14$ & $0(0)$ & $2(0.7)$ & $4(1.5)$ & $1(0.4)$ & $3(1.1)$ & $3(1.1)$ & $0(0)$ & $13(0.7)$ \\
\hline $15-19$ & $1(0.3)$ & $3(1)$ & $2(0.7)$ & $0(0)$ & $1(0.3)$ & $0(0)$ & $2(0.7)$ & $9(0.4)$ \\
\hline $20-24$ & $1(0.3)$ & $2(0.6)$ & $4(1.2)$ & $2(0.6)$ & $1(0.3)$ & $1(0.3)$ & $1(0.3)$ & $12(0.5)$ \\
\hline $25-29$ & $3(0.8)$ & $3(0.8)$ & $3(0.8)$ & $2(0.5)$ & $4(1.1)$ & $2(0.5)$ & $2(0.5)$ & $19(0.7)$ \\
\hline $30-34$ & $4(1.1)$ & $2(0.6)$ & $6(1.7)$ & $6(1.7)$ & $5(1.4)$ & $4(1.1)$ & $3(0.9)$ & $30(1.2)$ \\
\hline $35-39$ & $2(0.6)$ & $4(1.2)$ & $5(1.6)$ & $3(0.9)$ & $3(0.9)$ & $4(1.2)$ & $10(3.1)$ & $31(1.4)$ \\
\hline $40-44$ & $1(0.3)$ & $1(0.3)$ & $2(0.7)$ & $2(0.7)$ & $4(1.3)$ & $3(1)$ & $3(1)$ & $16(0.8)$ \\
\hline $45-49$ & $0(0)$ & $2(0.7)$ & $4(1.5)$ & $1(0.4)$ & $4(1.5)$ & $5(1.8)$ & $3(1.1)$ & $19(1.0)$ \\
\hline $50-54$ & $0(0)$ & $1(0.4)$ & $2(0.8)$ & $2(0.8)$ & $2(0.8)$ & $1(0.4)$ & $4(1.6)$ & $12(0.7)$ \\
\hline $55-59$ & $1(0.4)$ & $3(1.3)$ & $2(0.9)$ & $5(2.2)$ & $3(1.3)$ & $2(0.9)$ & $3(1.3)$ & $19(1.2)$ \\
\hline $60-64$ & $2(1.1)$ & $4(2.2)$ & $3(1.7)$ & $1(0.6)$ & $9(5)$ & $4(2.2)$ & $4(2.2)$ & $27(2.1)$ \\
\hline $65-69$ & $3(2.1)$ & $3(2.1)$ & $4(2.8)$ & $4(2.8)$ & $2(1.4)$ & $2(1.4)$ & $3(2.1)$ & $21(2.1)$ \\
\hline $70-74$ & $6(5)$ & $3(2.5)$ & $1(0.8)$ & $2(1.7)$ & $0(0)$ & $6(5)$ & $5(4.2)$ & $23(2.8)$ \\
\hline$\geq 75$ & $4(1.9)$ & $9(4.4)$ & $11(5.4)$ & $9(4.4)$ & $16(7.8)$ & $13(6.3)$ & $14(6.8)$ & $76(5.3)$ \\
\hline Unknown & $0(0)$ & $1(0)$ & $0(0)$ & $0(0)$ & $1(0)$ & $0(0)$ & $1(0)$ & $0(\mathrm{NA})$ \\
\hline Total & $35(0.8)$ & $49(1.2)$ & $61(1.4)$ & $57(1.3)$ & $70(1.7)$ & $60(1.4)$ & $68(1.6)$ & $400(1.3)$ \\
\hline
\end{tabular}

NA: not applicable. 
Information on iGAS isolate site and patient demographics were collected from 1 January 2004, while data on clinical presentation, risk factors, and outcomes were collected from 1 January 2005. Antimicrobial susceptibility results were collected from 1 January 2008. Typing of isolates was not routinely performed. We extracted and analysed data from 1 January 2004 to 31 December 2010.

Monthly, yearly and average annual incidence was calculated for males and females by five-year age groups using the matched age and sex population enumerated in 2006 census as the denominator [3].

A regression against time model was fit to test for trend in incidence of iGAS over time.

\section{Results}

Over the seven year period, 400 cases of iGAS ( 390 confirmed cases, five probable and five not specified) were notified. No iGAS clusters were notified over the period from 2005 to 2010, for which enhanced data were available. There was a significant increase in iGAS incidence from 2004: 0.8 per 100,000 population to 2008: 1.6 per 100,000 population $\left(R^{2}=0.85\right)$ and in 2009 and 2010 there was no further increase (Table 1). The highest incidence annually was between late December and late August but this was not statistically significant by individual year or when averaged over seven years ( $p>0.05$ ), hence seasonal trends could not be inferred by this study.

\section{Demographics}

A total of 205 males (average annual incidence of $1.38 / 100,000$ males) and 195 females (average annual incidence of $1.31 / 100,000$ females) were notified as cases (ratio: 1.05, 95\% Confidence Interval $(\mathrm{Cl})$ : $0.89-1.24)$.
Cases occurred in all age groups (median age: 44 years, range: 0-97) but children up to five years of age and adults aged 60 years and over had the highest age-specific incidence rates (Table 1). In 2010, there was also a peak in adults aged 35 to 39 (three of whom were intravenous drug users). No meaningful differences between average annual incidences for males versus females could be observed in any age group.

\section{Characteristics of Streptococcus pyogenes isolates}

Of the 390 confirmed cases with S. pyogenes isolated from a normally sterile site, the site/s from which the isolate was/were obtained was recorded in 225 cases. Of these, the most frequently recorded site was blood $(n=198,88 \%)$. S. pyogenes was also isolated from deep tissue $(n=15)$, joint $(n=3)$, abscess $(n=6)$, pleural fluid $(n=3), C S F(n=2)$, peritoneal fluid $(n=1)$, periorbital haematoma or tissue $(n=2)$, aspirate $(n=1)$, bone $(n=1)$, ventriculoperitoneal (VP) shunt tip $(n=1)$ and wounds $(n=2)$. S. pyogenes was isolated from more than one site in $11 \%$ of cases (25/225) with blood and another site being the most common combination.

Antimicrobial susceptibility data were available on 149 isolates between 2008 and 2010. Of isolates tested against each of the following, all were susceptible to penicillin $(n=147)$ and vancomycin $(n=117)$. Erythromycin resistance was reported in 13 of 140 (9.3\%) isolates; clindamycin resistance in two of 57 isolates and tetracycline resistance in seven of 69 isolates.

\section{Clinical presentation}

Clinical syndromes associated with iGAS were recorded in $44 \%(174 / 400)$ of cases. Bacteraemia was the most commonly recorded presentation $(n=115$, $66 \%)$, followed by cellulitis ( $n=70,40 \%)$, STSS $(n=28$, $16 \%)$, pneumonia $(n=27,16 \%)$, and necrotising fasciitis $(n=19,11 \%)$ (Table 2$)$. Some cases had more than

TABLE 2

Clinical presentation for cases of invasive group A streptococcal infection, Ireland, 2005-2010 (n=174)

\begin{tabular}{|l|c|c|c|} 
Clinical diagnosis & $\begin{array}{c}\text { Number of cases (\%) } \\
\mathrm{n}=174\end{array}$ & $\begin{array}{c}\text { Number of cases with STSS } \\
\text { diagnosis (\%) } \\
\mathrm{n}=28\end{array}$ & $\begin{array}{c}\text { Number of cases of bacteraemia } \\
\text { diagnosis (\%) } \\
\mathrm{n=115}\end{array}$ \\
\hline Bacteraemia & $115(66)$ & $21(75)$ & $115(100)$ \\
\hline Cellulitis & $70(40)$ & $8(29)$ & $21(36)$ \\
\hline Streptococcal toxic shock syndrome & $28(16)$ & $6(100)$ & $21(18)$ \\
\hline Pneumonia & $27(16)$ & $7(25)$ & $8(7)$ \\
\hline Necrotising fasciitis & $19(11)$ & $2(7)$ & $5(4)$ \\
\hline Septic arthritis & $11(6)$ & $1(4)$ & $3(3)$ \\
\hline Puerperal sepsis & $9(5)$ & $2(7)$ & $5(4)$ \\
\hline Myositis & $7(4)$ & $1(4)$ & $2(2)$ \\
\hline Meningitis & $5(3)$ & $1(4)$ & $1(1)$ \\
\hline Peritonitis & $3(2)$ & $1(4)$ & $1(1)$ \\
\hline Erysipelas & $1(1)$ & & \\
\hline
\end{tabular}

STSS: streptococcal toxic shock syndrome.

A case may have more than one clinical diagnosis recorded. 
one clinical presentation. In $66 \%(76 / 115)$ of patients with bacteraemia, at least one other presentation was also recorded, with cellulitis being the most common. In $89 \%(25 / 28)$ of cases where STSS was recorded, another presentation was also recorded, with bacteraemia being the most common, followed by cellulitis (Table 2).

\section{Underlying risk factors}

Of the 158 cases (158/400, 40\%), including nine deaths (9/11) where invasive group A streptococcal infection was main the cause, and for whom underlying risk factor information was reported, skin lesions or surgical wounds were the most frequently recorded $(n=58,37 \%)$. Intravenous drug use, steroid therapy, childbirth, diabetes mellitus, malignancy, nonsteroidal anti-inflammatory drug (NSAID) use, alcohol abuse and varicella infection were recorded as underlying risk factor in less than one percent to $13 \%$ of cases (Table 3 ).

\section{Mortality}

Over the seven year period, outcome data at seven days were provided for 141 of 400 cases (35\%). Sixteen deaths occurred; iGAS was identified as the main cause of death for eleven cases (seven-day case fatality rate (CFR), $8 \%$ ) and as a contributory cause of death for three cases. For the two remaining cases, it was not specified whether iGAS was the main or a contributory cause of death. The majority of deaths occurred in people aged 65 years and over (nine of 16). There were equal numbers of deaths in men and women. Of the cases with STSS where outcome was recorded, six deaths occurred giving a CFR of $21 \%(6 / 28)$. Of the 11 deaths directly attributable to iGAS (seven females, four males), six occurred in people aged 65 years of age and over. Risk factor data were available for nine of the eleven deaths where iGAS was the main cause. Skin lesions or surgical wounds and NSAID use were

\section{TABLE 3}

Underlying risk factors for cases of invasive group A streptococcal infection, including nine deaths at seven days, where invasive group A streptococcal infection was the main cause, Ireland, 2005-2010 ( $\mathrm{n}=158)$

\begin{tabular}{|l|c|c|}
\hline Underlying risk factor & $\begin{array}{c}\text { Number of } \\
\text { cases }(\%) \\
\mathrm{n}=158\end{array}$ & $\begin{array}{c}\text { Number of } \\
\text { deaths } \\
\mathrm{n}=9\end{array}$ \\
\hline Skin lesion/surgical wound & $58(37)$ & 3 \\
\hline Intravenous drug use & $20(13)$ & 2 \\
\hline Malignancy & $18(11)$ & 1 \\
\hline Diabetes & $17(11)$ & 1 \\
\hline Childbirth & $15(9)$ & 0 \\
\hline Steroids & $11(7)$ & 1 \\
\hline Nonsteroidal anti-inflammatory drugs & $10(6)$ & 3 \\
\hline Alcohol abuse & $8(5)$ & 0 \\
\hline Varicella & $1(1)$ & 0 \\
\hline No identified risk factor & $39(25)$ & 3 \\
\hline
\end{tabular}

Cases could have more than one risk factor. the most commonly recorded risk factor, with small numbers of other risk factors recorded (Table 3 ). Clinical presentation was recorded for 10 of the 11 cases, with two or more clinical syndromes recorded in seven cases. Six presented with bacteraemia (an additional four had GAS isolated from their blood), four with STSS, three with pneumonia, three with myositis, two with necrotising fasciitis and two with cellulitis.

\section{Discussion}

This study provides an overview of the first seven years of iGAS surveillance in Ireland. The incidence of iGAS increased from 0.8 per 100,000 population in 2004 to 1.6 in 2008, after which it stabilised, with the highest age-specific incidence rates seen in children less than five years of age and adults aged 60 years and over. Like other countries, there appears to be slightly more men than women infected and more cases occurring in late winter/spring, however, these patterns are not statistically significant, which may be due to small numbers or some other unknown factor. Eleven people died with iGAS identified as the main cause of death (sevenday case fatality rate, $8 \%$ ). Most deaths occurred in people aged 65 years and over.

The incidence of iGAS reported in Ireland was lower than that reported in the United Kingdom (UK), Nordic countries and North America but higher than southern and eastern European countries [4-14]. In 2009, the rate of GAS bacteraemia for England, Wales and Northern Ireland was 2.6 per 100,000 population, with regional rates ranging from 2.1 in the East Midlands to 3.2 in the North (UK figures here only include data from bacteraemia) [13]. In the United States (US) in 2009, the estimated rate of iGAS disease was 3.6 per 100,000 population [12] (normally sterile site or wound plus NF or STSS). In Sweden and Finland, the incidence rates in 2010 were 3.8 and 3.3 per 100,000 , respectively (Sweden: no definition on website; Finland: S. pyogenes isolated from blood or cerebrospinal fluid) $[5,10]$. The clinical presentations of iGAS in Ireland were similar to that reported in other countries $[6,7,15,16]$, however mortality due to iGAS was lower than that found in the Strep-EURO study [6], though similar to the North American continent [7-9,15].

It is difficult to be certain what accounts for these differences in iGAS incidence. It is possible that there is a real difference in incidence rates due to some unknown environmental reason, such as population density or climate. It is more likely that empiric antibiotic prescribing practices in Ireland, which are relatively high, may account for some of the difference between Ireland and elsewhere. For example, in 2008, outpatient penicillin use was higher in Ireland at 11.34 Defined Daily Dose (DDD) per 1,000 inhabitants per day than Finland at 6.11 DDD, Sweden at 7.37 DDD and the UK 7.95 DDD per 1,000 inhabitants per day [17].

However, it may be the case that difference in incidence of iGAS in Ireland is due to notification rather than a 
real difference in incidence. It is also likely that as a newly notifiable disease, not all practitioners notified cases of iGAS initially. There was an increase in notifications between 2006 and 2008, followed by a stabilising of rates in 2009 and 2010, which indicates that at least some of the difference in incidence in Ireland compared with other countries was due to practice with respect to notifications. In addition, the number of microbiology laboratories using the national electronic infectious disease reporting system, CIDR, increased over the study period. While every effort was made to collect the same data by other methods from laboratories not on CIDR, it is possible that some data from these laboratories were not collected. As more laboratories came online, this may have accounted for some of the observed increase.

A small number of cases may go unreported as a result of Ireland's current case definition, where confirmed cases of iGAS include only cases where $S$. pyogenes is isolated from a normally sterile site and probable cases include only patients presenting with STSS and S. pyogenes isolated from a non-sterile site. Other countries have broader case definitions. In the StrepEURO study confirmed cases included patients with S. pyogenes isolated from a normally sterile site, or non-sterile site in combination with clinical signs of STSS [6]. The UK case definition includes patients with non-sterile site isolates with one of the following severe presentations: pneumonia, necrotising fasciitis, puerperal sepsis, meningitis or septic arthritis [13] and the US case definition includes patients with a wound culture accompanied by necrotising fasciitis or STSS [12]. This may lead to a probably very small number of cases (e.g. post-varicella iGAS with necrotising fasciitis and $S$. pyogenes cultured from wound swab) not being counted in Ireland that would be counted in other countries.

Microbiological findings confirm that iGAS remains susceptible to penicillin and that penicillin should continue to be the first line treatment where iGAS is suspected.

Our study has a number of limitations. Of the 244 enhanced surveillance forms completed between January 2005 and December 2010 (representing 68\% of cases over this period, $n=359$ ), $94 \%$ contained data on the site of the isolate, $71 \%$ on the clinical presentation and $65 \%$ on risk factors. It is possible that the cases on which more complete data were obtained were not representative of the full dataset. This may impact on data validity and introduce bias.

A further limitation is lack of data on emm/M-protein gene types. Certain emm/M-types of $S$. pyogenes are known to be more virulent than others, e.g. emm 1 and $3[7,15]$. However, as there is no Irish streptococcal reference laboratory, no typing data were collated nationally to investigate whether more virulent emm/M-types were associated with more severe disease or whether there was any change in iGAS types circulating over time.

In conclusion, the addition of iGAS to the list of notifiable diseases in Ireland has yielded useful information in the understanding of iGAS in Ireland. However, there continue to be constraints, most notably the absence of a national reference laboratory, the incomplete information collected on enhanced surveillance data and the lack of a universal or at least European standardised case definition for iGAS.

The authors recommend continued collection of enhanced surveillance data on cases of iGAS and efforts to improve completeness of data collected on enhanced surveillance forms. We also recommend an international review of the case definition with a view to the establishment of a consistent case definition across all countries, thereby enabling standardised international comparisons. Finally, the authors believe that the establishment of a national streptococcal reference laboratory is essential to enable a better understanding of iGAS in Ireland.

\section{Acknowledgments}

Special thanks are due to the departments of public health and microbiology laboratories who collected the data used to inform this study.

\section{References}

1. Invasive Group A Streptococcus Sub-Committee. The management of invasive group A streptococcal infections in Ireland: Health Protection Surveillance Centre; 2006. [Accessed 10 Oct 2011]. Available from: http://www.hpsc. ie/hpsc/A-Z/Other/GroupAStreptococcalDiseaseGAS/ Publications/File,2080,en.pdf

2. Health Protection Surveillance Centre (HSPC). Annual Report 2005. Invasive Group A Streptococcal Disease in Ireland, 2005: HSPC; 2006. ISSN 1649-0436. Available from: http://www. hpsc.ie/hpsc/A-Z/Other/GroupAStreptococcalDiseaseGAS/ Publications/AnnualReports/File,2689,en.pdf

3. Central; Statistics Office. Census 2006. Principal Demographic Results. Dublin: Stationery Office; Mar 2007. Available from: http://www.cso.ie/census/documents/Amended\%20Final\%20 Principal\%20Demographic\%20Results\%202006.pdf

4. Lamagni T. The epidemiology of severe streptococcus pyogenes disease in Europe. Helsinki: National Public Health Institute; 2008. [Accessed 10 Oct 2011] Available from: https://helda.helsinki.fi/bitstream/handle/10138/20461/ theepide.pdf?sequence $=1$

5. National institute for Health and Welfare Finland. Streptococcus pyogenes. 2010. [Accessed 10 Oct 2011]. Available from: http://www3.ktl.fi/stat/

6. Lamagni T, Darenberg J, Luca-Harari B, Siljander T, Efstratiou A, Henriques-Normark B, et al. Epidemiology of Severe Streptococcus pyogenes Disease in Europe. J Clin Microbiol. 2008;46(7):2359-67.

7. Tyrrell G, Lovgren M, Kress B, Grimsrund K. Invasive Group A streptococcal disease in Alberta, Canada (2000-2002). J Clin Microbiol. 2005;43(4):1678-83

8. Veasy L, Wiedmeier S, Orsmond G, Ruttenberg H, Boucek $M$, Roth S. Resurgence of acute rheumatic fever in the intermountain area of the United States. N Engl J Med. 1987;316(8):421-7.

9. Demers B, Simor A, Vellend H, Schlievert P, Byrne S, Jamieson F. Severe invasive group A streptococcal infections in Ontario, Canada. Clin Infect Dis. 1993;16(6):792-800.

10. Smittskyddsinstitutet. Beta hemolytic group-Astreptococci (GAS) (invasiv infection). [Accessed 10 Oct 2011]. Available from: http://www. 
smittskyddsinstitutet.se/in-english/statistics/

beta-hemolytic-group-a-streptococci-gas-invasiv-infection/

11. Lamagni T, Efstratiou A, Vuopio-Varkila J, Jasir A,

Schalén C. The epidemiology of severe Streptococcus pyogenes associated disease in Europe. Euro Surveill. 2005;10(9): pii=563. Available from: http://www.

eurosurveillance.org/ViewArticle.aspx?Articleld $=563$

12. Emerging Infections Program Network (EIPN), Group A

Streptococcus. Active Bacterial Core Surveillance Report.

EIPN, Group A Streptococcus: Centers for Disease Control and Prevention; 2009. [Accessed 10 Oct 2011]. Available from: http://www.cdc.gov/abcs/reports-findings/survreports/gaso8. pdf

13. Health Protection Agency. Infection reports. Pyogenic and non-pyogenic streptococcal bacteraemia, England, Wales and Northern Ireland: 2009. Health Protection Report 2009:4(46) - 19 November 2009. Available from: http://www.hpa.org.uk/ web/HPAwebFile/HPAweb_C/1287144931510

14. Lamagni T, Neal S, Keshishian C, Alhaddad N, George $R$, Duckworth G, et al. Severe Streptococcus pyogenes infections, United Kingdom, 2003-2004. Emerg Infect Dis. 2008;14(2):202-9.

15. O'Loughlin RE, Roberson A, Cieslak PR, Lynfield R, Gershman $\mathrm{K}$, Craig A, et al. The epidemiology of invasive group $\mathrm{A}$ streptococcal infection and potential vaccine implications: United States, 2000-2004. Clin Infect Dis. 2007;45:853-62.

16. O'Grady KA, Kelpie L, Andrews RM, Curtis N, Nolan TM, Selvaraj G, et al. The epidemiology of invasive group $A$ streptococcal disease in Victoria, Australia. Med J Aust. 2007;186:565-9.

17. European Surveillance of Antimicrobial Consumption (ESAC). ESAC yearbook 2008; ESAC; 2008. pg 33. [Accessed 10 Oct 2011]. Available from: http://www.esac.ua.ac.be/main. aspx?c $={ }^{\star} E S A C 2 \& n=50036$ 